Pirineos, 147-148: 81 a 96, JACA; 1996

\title{
RELACIONES SUELO-PLANTA-HERBIVORO EN UN SISTEMA PASTORAL DE MONTAÑA
}

\author{
I. Alonso1, C. Garcia-Olalla ${ }^{2}$ \\ ${ }^{1}$ Institute of Terrestrial Ecology. Banchory Research Station. Banchory, AB31 4BY, Escocia,Gran Bretaña \\ 2 Dpto. Bioquímica y Biologia Molecular. Universidad de León. 24071 León. España
}

RESUMEN.- Se analizaron las características edáficas de seis comunidades de interés pastoral en la montaña de León denominadas en función de la especie más representativa (Nardus stricta, Bromus erectus, Genista florida, Erica australis, Genista occidentalis, Calluna vulgaris), así como las concentraciones minerales de $\mathrm{Ca}, \mathrm{Cu}, \mathrm{Fe}, \mathrm{Mg}, \mathrm{N}, \mathrm{K}$ y $\mathrm{Na}$ en suelos y pastos por su influencia sobre las características nutritivas de la dieta de los herbívoros. Según los resultados obtenidos, los suelos presentaron en general unas buenas condiciones para el desarrollo vegetal. No se apreció ninguna relación entre la concentración de minerales en suelos y plantas. Las cantidades de $\mathrm{Ca}$ y $\mathrm{Cu}$ presentes en la vegetación fueron insuficientes para el mantenimiento de los herbívoros domésticos al menos durante parte de su estancia en el puerto. Las concentraciones minerales en la vegetación sufrieron variaciones estacionales debido a procesos de madurez y cambios fisiológicos, en mayor o menor medida según el tipo de planta y el ambiente en que se encuentre.

ABSTRACT.- The soil characteristics of six communities with pastoral interest in León mountain (Spain) and the $\mathrm{Ca}, \mathrm{Cu}, \mathrm{Fe}, \mathrm{Mg}, \mathrm{N}, \mathrm{K}$ and Na contents of soils and vegetation were analysed in relation with the nutritional characteristics of herbivores' diet. The communities were named after the most representative species: Nardus stricta, Bromus erectus, Genista florida, Erica australis, Genista occidentalis and Calluna vulgaris. Soils showed in general good conditions for vegetal growth. There were no relationships between soil and plants mineral contents. $\mathrm{Ca}$ and $\mathrm{Cu}$ were deficient in some moments during the stay of the animals in the mountain. Mineral concentrations in vegetation communities varied seasonally due to maturity processes and physiological changes in plants, depending on the kind of plant and the microenvironment where they grew.

ZUSAMMENFASSUNG.-Die Bodeneigenschaften von sechs Pflanzengesellschaften mit Weidefähigkeit aus dem Bergen von León (Spanien) wurden untersucht, und die Analysergebnisse der Boden- und Pflanzen-konzentrationen von $\mathrm{Ca}, \mathrm{Cu}$, 
$\mathrm{Fe}, \mathrm{Mg}, \mathrm{N}, \mathrm{K}$ und Na präsentiert. Die Gesellschaften sind nach ihren dominanten Spezies benannt: Nardus stricta, Bromus erectus, Genista florida, Erica australis, Genista occidentalis und Calluna vulgaris. Wir konnten feststellen, da $\beta$ die Boden gute Bedingungen für das Pflanzenwachstum besitzen. Es bestand kein Zusammenhang zwischen der mineralischen Zusammensetzung der Böden und die der Pflanzen. Außerdem wurde beobachtet, daß die Mengen an $\mathrm{Ca}$ und $\mathrm{Cu}$, die in der Vegetationszeit zur Verfügung stehen, nicht ausreichend sind um Pflanzenfresser zu versogen. Die mineralische Zusammensetzung in den Pflanzengesellschaften veränderte sich saisonbedingt auf Grund des Reifeprozesses und auf Grund physiologischer Veränderungen. Diese veränderungen waren abhangig von den Pflanzenarten und deren Mikroumgebung .

Keywords: Mountain grasslands, soil characteristics, soil and plant mineral composition, seasonality, herbivore diet.

\section{Introducción}

E1 $50 \%$ de la provincia de León tiene una altitud mayor de $1.000 \mathrm{~m}$ y el 25 $\%$ sobrepasa los $1.200 \mathrm{~m}$ pero apenas existen datos básicos de carácter productivo sobre los pastos de montaña, aun siendo éstos no sólo un tipo de ecosistema de gran extensión en la provincia, sino que junto con los prados de siega suponen la base productiva de una de las principales áreas del sector primario: la ganadería.

Además de las características bromatológicas, en la valoración de los pastos como base de la producción ganadera, también es importante conocer su composición mineral. Aunque la mayoría de los trabajos hacen hincapié en que las hojas jóvenes tienen más proteína y menos fibra, éstas tienen también menor concentración de minerales (EGAN, 1975). La deficiencia o exceso de elementos minerales puede afectar al desarrollo del ganado y por ello, el estudio geoquímico del suelo o el análisis detallado del crecimiento de las plantas puede ser de gran ayuda a la hora de predecir o detectar insuficiencias minerales en los rebaños.

La disponibilidad de los minerales en el suelo es el más importante, pero no el único, de la serie de factores que afectan a la concentración de esos elementos en las plantas. El segundo factor en importancia es el tipo de planta de que se trate y la cantidad de nutrientes minerales necesaria para su crecimiento, salud y reproducción (BEESON y MATRONE, 1976). La concentración de un determinado elemento en un vegetal depende así mismo de su estado de madurez [aunque aparecen también fluctuaciones anuales PEGTEL (1987)], de las condiciones ambientales en que se desarrolle (MONTAL-

- VO et al., 1975) y de la parte de la planta que se analice (las raíces tienen mayor concentración de elementos minerales). 
Los elementos minerales del suelo no son absorbidos por las plantas en su totalidad, pero su análisis sirve para determinar la disponibilidad y cuáles son las condiciones en las que la vegetación está creciendo. Los niveles en que se encuentran en los suelos determinan la abundancia y la distribución de las plantas (BEN-SHAHAR y COE, 1992), características que influyen en la distribución y comportamiento alimenticio de los herbívoros (REVESADO et al., 1994). Por ello, la cantidad total de los elementos minerales en las plantas es un indicador muy útil de la cantidad disponible para los animales, aunque sólo se utilice una pequeña fracción (BURRIDGE, 1987).

La climatología y orografía propias de estos pastos limita su utilización a los meses de verano, pero a pesar de la brevedad del periodo considerado existen variaciones en la calidad y cantidad de la oferta forrajera (ALONSO, 1994; REVESADO et al., 1994; ALONSO y GARCIA-OLALLA, 1997). Debido a la escasez de este tipo de datos sobre los pastos leoneses, los objetivos que se plantearon en este trabajo fueron: a) estudiar las características de los suelos y las comunidades de pastos de un puerto de montaña para evaluar el status nutritivo tanto de suelos para la producción vegetal como de plantas para la producción animal; b) determinar las variaciones estacionales en las concentraciones minerales en las comunidades vegetales, cuantificando las diferencias; c) interrelacionar toda la información obtenida para llegar a la comprensión global del sistema y poder decidir las bases de su manejo.

El atractivo de la zona objeto de este estudio se basa en su condición de sistema representativo de la Cordillera Cantábrica, tanto a nivel de su vegetación, por su diversidad, como por el manejo a que ha sido sometido durante siglos. Las comunidades vegetales presentes se caracterizan por su amplia distribución, algunas a nivel europeo.

\section{Material y métodos}

\subsection{Zona de estudio}

El sistema pastoral elegido para este estudio se localiza en el Puerto de San Isidro, en la Cordillera Cantábrica (provincia de León). En este área, se seleccionó un puerto por sus características fisionómicas, físicas, botánicas y de uso tradicional. Un puerto en este contexto es una superficie de terreno gestionada por la autoridad local y que se alquila como pasto a los pastores de rebaños migratorios y a otros ganaderos. El área tiene una altitud que varía entre 1.400 y $1.600 \mathrm{~m}$ y comprende aproximadamente 100 hectáreas de terreno caracterizado por la presencia de substratos ácidos y básicos. El puerto ha sido pastado desde antiguo por rebaños de ovejas merinas trashuman- 
tes, así como por ganado vacuno y caballar de zonas cercanas. La vegetación presente en el área está muy influenciada por este pastoreo extensivo secular, como en la mayoría de las zonas de montaña. El pasto se engloba principalmente en seis tipos de vegetación definidos por parámetros fisionómicos (dominancia de ciertas formas de vida y de crecimiento), fitoecológicos (abundancia de determinadas especies), agronómicos (valor pastoral) y de distribución por el puerto en función de las características edáficas y de la presión de pastoreo a que han sido sometidas (ALONSO, 1994).

\subsection{Análisis de los suelos}

Para llevar a cabo este trabajo, en 1990 se tomaron veinte muestras al azar de suelo de cada una de las comunidades vegetales identificadas y denominadas en función de las especies representativas. Éstas fueron: 1.Pastos con Nardus stricta, 2.- Pastos con Bromus erectus, 3.- Pastos con Genista florida, 4.- Pastos con Erica australis, 5.- Pastos con Genista occidentalis, 6.- Pastos con Calluna vulgaris. Las muestras correspondían a los 5-20 primeros $\mathrm{cm}$ de profundidad, por ser ésta la zona donde se encuentran la mayor parte de las raíces. Previamente se retiró el tapiz vegetal y el humus. Las muestras se secaron a temperatura ambiente en el laboratorio y fueron tamizadas con una malla de $2 \mathrm{~mm}$ de poro con la que se separaron las partículas gruesas.

Para la descripción de los suelos de la zona de estudio se han considerado tres apartados:

1) Las características físicas del suelo: humedad (relación en porcentaje entre el peso inicial y peso después de secar las muestras a $105^{\circ} \mathrm{C}$ durante 24 horas), capacidad de retención (medida de la fase de drenaje rápido, embebiendo las muestras en agua y midiendo el incremento de peso respecto a la muestra seca a las 24 horas) y granulometría (\% de arcilla, de limo y de arena). Antes de realizar el análisis granulométrico, la materia orgánica de la muestra fue eliminada por calcinación, mediante un ataque con agua oxigenada. La técnica seguida para determinar la composición granulométrica es la descrita por la "International Soil Science Society", basada en el método de Bouyoucos (ALLEN, 1989). Los datos obtenidos con este tipo de análisis se suelen disponer en un triángulo equilátero.

2) Las características químicas del suelo: $\mathrm{pH}$ en agua y materia orgánica, analizadas según las técnicas descritas por ALLEN (1989). La muestra debe estar previamente desecada a $105^{\circ} \mathrm{C}$ por lo que se utilizan las mismas que en la determinación de la humedad.

3) La fracción mineral. El análisis mineralógico de las 120 muestras de suelo ( 6 comunidades $\times 20$ muestras) se realizó con espectrofotometría de absorción atómica, utilizándose un aparato de la marca Perkin Elmer Modelo 11003. Se 


\section{SUELO-PLANTA-HERBÍVORO EN LEÓN}

analizaron los macronutrientes $\mathrm{Ca}, \mathrm{Mg}, \mathrm{N}, \mathrm{K}$ y los micronutrientes $\mathrm{Cu}, \mathrm{Fe}$ y $\mathrm{Na}$ siguiendo las técnicas descritas por los fabricantes. La cantidad de nitrógeno orgánico presente en las muestras se determinó mediante el método Kjeldahl.

\subsection{Análisis de los pastos}

En el mismo año se cortaron a mano 10 muestras seleccionadas al azar de cada pasto cada mes a lo largo de la estación de pastoreo (junio, julio, agosto y septiembre), que fueron secadas a $65^{\circ} \mathrm{C}$ durante 72 horas y posteriormente molidas, utilizando una malla de $1 \mathrm{~mm}$ de diámetro. Las 240 muestras de las comunidades vegetales presentes en San Isidro (6 comunidades $x 10$ muestras x 4 meses) fueron analizadas siguiendo la técnica descrita por DE RUIG (1986).

\subsection{Análisis estadístico}

Los resultados obtenidos de los análisis correspondientes para cada elemento fueron comparados mediante análisis de varianza con unidades repetidas, de una vía para los elementos en el suelo en las seis comunidades y de dos vías para los elementos en la vegetación considerando las variaciones estacionales. La significación de las medias se comprobó mediante el test de Tukey por ser muestras de igual tamaño.

\section{Resultados}

En la tabla 1 se indican las características físicas (porcentajes medios de los elementos granulométricos, humedad y capacidad de retención) y químicas ( $\mathrm{pH}$ en agua y porcentaje de la materia orgánica) de los suelos de cada comunidad. Hay que tener en cuenta que cuando el porcentaje de materia orgánica es alto, como fue el caso en todas las comunidades, los análisis granulométricos tienen un rango de variación mayor.

La figura 1 representa la localización de los suelos de las seis comunidades estudiadas en un triángulo de texturas. Tres de las comunidades (Genista florida, Erica australis y Calluna vulgaris) se encuentran sobre suelos prácticamente francos, es decir, suelos equilibrados y con buenas propiedades para el desarrollo vegetal.

Los suelos más equilibrados en cuanto a los elementos minerales analizados fueron los de las comunidades con Bromus erectus y Genista occidentalis, que presentaron, como cabría esperar por su situación sobre sustrato calizo, las concentraciones de calcio más altas, difiriendo significativamente del resto (tabla 2). Las comunidades con especies de la familia Ericaceae (Erica 
australis y Calluna vulgaris) presentaron valores relativamente bajos de Ca, $\mathrm{Cu}, \mathrm{K}, \mathrm{Mn}$ y $\mathrm{Na}$ en el suelo. Los suelos de la comunidad de Calluna vulgaris resultaron ser los más pobres para todos los elementos.

La evolución de la concentración de cada elemento en la vegetación a lo largo de la estación de pastoreo queda reflejada en la figura 2. La tabla 3 complementa a la figura 2 y es una síntesis de los análisis realizados. En ella se indican las medias de los valores por comunidades y por meses para cada elemento, así como la significación de las diferencias existentes. No hubo un patrón general, sino que aparecieron tres tendencias generales:

1.- Elementos cuya concentración disminuye de primavera a verano y se recupera en otoño ( $\mathrm{Cu}, \mathrm{Mg}$-excepto en Bromus erectus y Genista florida-, $\mathrm{N}, \mathrm{K}$ );

2.- Elementos con una concentración relativamente alta durante el verano (Ca, Mg en Bromus erectus);

3.- Elementos con gran variabilidad (Fe, $\mathrm{Na})$.

\section{Discusión}

\subsection{Características físicas del suelo}

Las comunidades de Nardus stricta y Genista florida presentaron los suelos más diversos granulométricamente, como indican las desviaciones estándar de los valores obtenidos, mayores que en otras comunidades. Los pastos con Erica australis y Bromus erectus, en cambio, se asientan sobre suelos más homogéneos en cuanto al tamaño de las partículas. Los suelos de las comunidades de Nardus stricta, Erica australis, Calluna vulgaris y Genista florida, es decir, los que se asientan sobre sustrato silíceo, son los más similares entre sí, presentando diferencias no significativas en la mayoría de los casos. Los suelos de los pastos con Bromus erectus y Genista occidentalis difieren significativamente del otro grupo, y son distintos entre sí en la proporción de arena.

Si se comparan estos datos con los obienidos para los suelos de otros tipos de pastos de montaña (GARCIA et al., 1988), podemos observar que el rango de variación en nuestro caso es muy grande y sólo estarían dentro de la misma categoría las proporciones de limo. Se ha encontrado mayor afinidad en este sentido con los suelos forestales de la Sierra de Gata (GALLARDO y CUADRADO, 1980). Los suelos de las comunidades con brezo presentaron las proporciones de limo más elevadas.

Los substratos de textura franca tienen en general una buena capacidad de retención del agua, independientemente de la proporción de materia orgánica, a excepción de los suelos con Erica australis (tabla 1). Los suelos calizos (comunidades con Bromus erectus y con Genista occidentalis) se caracterizan 
SUELO-PLANTA-HERBÍVORO EN LEÓN

\begin{tabular}{lcccccc}
\cline { 2 - 6 } & E. australis & G. florida & N. stricta & C. vulgaris & B. erectus & G. occident. \\
\cline { 2 - 7 } & & & & & & \\
Arcilla (\%) & $9.16 \pm 3.18^{\mathrm{a}}$ & $14.38 \pm 4.62^{\mathrm{b}}$ & $8.62 \pm 3.64^{\mathrm{a}}$ & $9.55 \pm 2.43^{\mathrm{a}}$ & trazas $^{\mathrm{c}}$ & $1.54 \pm 1.42^{\mathrm{c}}$ \\
Limo (\%) & $44.27 \pm 4.02^{\mathrm{ab}}$ & $41.93 \pm 10.52^{\mathrm{ab}}$ & $32.84 \pm 10.95^{\mathrm{a}}$ & $47.92 \pm 6.60^{\mathrm{b}}$ & $12.80 \pm 5.03^{\mathrm{c}}$ & $24.00 \pm 9.11^{\mathrm{cd}}$ \\
Arena (\%) & $46.56 \pm 4.4^{\mathrm{ab}}$ & $43.69 \pm 10.21^{\mathrm{a}}$ & $58.54 \pm 12.83$ & $42.53 \pm 6.24^{\mathrm{a}}$ & $87.50 \pm 5.09^{\mathrm{c}}$ & $74.46 \pm 9.79^{\mathrm{d}}$ \\
pH en agua & $4.41 \pm 0.34^{\mathrm{ab}}$ & $3.99 \pm 0.12^{\mathrm{a}}$ & $4.69 \pm 0, .32^{\mathrm{b}}$ & $4.66 \pm 0, .35$ & $6.23 \pm 0.80^{\mathrm{c}}$ & $7.15 \pm 0.44^{\mathrm{d}}$ \\
M.O. (\%) & $16.70 \pm 2.57$ & $21.79 \pm 2.88^{\mathrm{bc}}$ & $15.95 \pm 1.14^{\mathrm{a}}$ & $16.60 \pm 1.40^{\mathrm{ab}}$ & $26.69 \pm 8.11^{\mathrm{c}}$ & $14.44 \pm 2.04^{\mathrm{a}}$ \\
Humedad (\%) & $3.48 \pm 0.32^{\mathrm{ab}}$ & $4.59 \pm 0.41^{\mathrm{bc}}$ & $3.51 \pm 0.40^{\mathrm{ab}}$ & $3.63 \pm 0.44^{\mathrm{ab}}$ & $5.41 \pm 2.02^{\mathrm{c}}$ & $2.76 \pm 0.39^{\mathrm{a}}$ \\
Cap. retención & $164.37 \pm 6.69^{\mathrm{a}}$ & $186.11 \pm 32.40^{\mathrm{ab}}$ & $185.11 \pm 9.81^{\mathrm{ab}}$ & $197.21 \pm 6.10^{\mathrm{b}}$ & $168.88 \pm 11.64^{\mathrm{a}}$ & $165.39 \pm 9.54^{\mathrm{a}}$
\end{tabular}

Tabla 1. Valores medios y desviación estándar de los parámetros edáficos de cada comunidad. Los valores en la misma línea seguidos por distinta letra son significativamente diferentes $(\mathrm{p}<0.05)$ según el test de Tukey: M.O. = materia orgánica. $n=20$.

Mean values and standard deviation of soil parameters in each community. Values on the same line with different superscripts are significatively different $(\mathrm{p}<0.05)$ using Tukey test. M.O. $=$ organic matter. $\mathrm{n}=20$.

ELEMENTOS

\begin{tabular}{|c|c|c|c|c|c|c|c|}
\hline & $\mathrm{Ca}$ & $\mathrm{Cu}$ & $\mathrm{Fe}$ & $\mathrm{Mg}$ & $\mathrm{N}$ & $\mathbf{K}$ & $\mathrm{Na}$ \\
\hline E. australis & 517. b & $24.0 \mathrm{~b}$ & 25435 a & $3646 \mathrm{c}$ & $0.62 \mathrm{bc}$ & $1295 \mathrm{bc}$ & $527 \mathrm{~b}$ \\
\hline B. erectus & 5481 a & 43.0 a & $21506 \mathrm{ab}$ & 2394 a & $1.10 \mathrm{a}$ & 3361 a & 2429 a \\
\hline G. florida & $602 b$ & $28.4 \mathrm{bc}$ & $23185 \quad a$ & $1259 \mathrm{~b}$ & $0.75 \mathrm{~b}$ & $1237 \mathrm{bc}$ & $1564 \mathrm{ab}$ \\
\hline N. stricta & $749 \mathrm{~b}$ & $37.4 \mathrm{ad}$ & $21795 \mathrm{ab}$ & $1823 \mathrm{ab}$ & $0.70 \mathrm{bc}$ & $2150 \mathrm{~d}$ & $1500 \mathrm{ab}$ \\
\hline G. occidentalis & 8226 a & $33.0 \mathrm{~cd}$ & $18442 \mathrm{ab}$ & $6099 \mathrm{~d}$ & $0.59 \mathrm{bc}$ & $1664 \mathrm{~b}$ & $801 \mathrm{~b}$ \\
\hline C. vulgaris & $420 \mathrm{~b}$ & $23.1 \quad b$ & $12758 \mathrm{~b}$ & $912 \mathrm{~b}$ & $0.54 \mathrm{c}$ & $1136 \mathrm{c}$ & $517 \mathrm{~b}$ \\
\hline E.S. & 850.85 & 2.06 & 2339.23 & 247.95 & 0.04 & 110.23 & 303.80 \\
\hline SIG & $* * *$ & $* * *$ & $* * *$ & $* * *$ & $* * *$ & $* * *$ & $* * *$ \\
\hline
\end{tabular}

Nivel de significación: ${ }^{* *}=\mathrm{P}<0.001 ;{ }^{* *}=\mathrm{P}<0.01 ; \mathrm{N} . S .=$ no significativo

Tabla 2. Análisis de la varianza de los valores medios de la concentración de cada uno de los elementos analizados en las muestras de suelo. E.S. = error estándar. SIG = significación. $\mathrm{n}=20$. ANOVA of mean values of the concentration of each analysed element in soil samples. E.S. = standard error. $\mathrm{SIG}=$ significance. $\mathrm{n}=20$. 
por una mayor pedregosidad, lo que supone una proporción muy baja de arcilla por el lavado de elementos finos (GARCIA et al., 1988). En este caso, el elevado porcentaje de materia orgánica en la comunidad de Bromus erectus contribuye a contrarrestar la tendencia común a la aridez de los suelos calizos (DUCHAUFOUR, 1975) (tabla 1). La comunidad de Nardus stricta ocupa suelos franco-arenosos, intermedios entre los otros dos grupos, con baja materia orgánica y alta capacidad de retención.

\subsection{Características químicas del suelo}

Los valores de $\mathrm{pH}$ de los suelos dependen sobremanera de la naturaleza química de la roca subyacente (KINZEL, 1983) y la climatología. El pH a su vez condiciona la disponibilidad de nutrientes para las plantas, ya que este parámetro está relacionado de forma inversa con su solubilidad. El suelo de la comunidad de Bromus erectus, con un $\mathrm{pH}$ intermedio entre el de Genista occidentalis (básico) y los del resto (ácido) (tabla 1) aparece en zonas de menor pendiente que el de Genista occidentalis. La suavidad del relieve favorece la formación de suelo de mejor calidad y el depósito de materia orgánica. Todo ello altera el $\mathrm{pH}$ inicialmente más básico de la roca madre caliza. Los valores de menor $\mathrm{pH}$ en ambas comunidades sobre sustrato calizo se encuentran en las zonas de ecotonía con los pastos de Nardus stricta.

La distribución de la materia orgánica a lo largo del perfil de la mayoría de los suelos es irregular, ya que se concentra en las capas superiores y disminuye con la profundidad (DAVIS et al., 1992). Su importancia se debe a que es una fuente de nutrientes para las plantas, determinando su producción, al tiempo que contribuye a estabilizar la estructura edáfica favoreciendo la retención de agua y la asimilación de otros nutrientes. Los valores de materia orgánica fueron significativamente más bajos en las comunidades de Genista occidentalis y Nardus stricta, respecto al resto. En el suelo de Bromus erectus, que presentó el valor más alto entre todas las comunidades, se distribuye de forma desigual entre las muestras. La causa podría estar en que la particular disposición de las piedras y fracciones gruesas del suelo crean microhábitats de distintas características. Estas condiciones influyen sobre la aparición y el ciclo de vida de los terófitos, cuya fase vegetativa en primavera o verano es corta y que pasan el resto del año enterrados en forma de semilla (ALONSO y GARCIA, 1993; ALONSO et al., 1993; ALONSO, 1994). La descomposición de la parte vegetativa proporciona una ligera acidez al suelo (GARCIA et al., 1992) lo que, como ya se indicó anteriormente, favorece la asimilación de nutrientes y el desarrollo de microorganismos del suelo. La carencia de la fracción fina en la comunidad de Bromus erectus (tabla 1), fracción que influye, entre otras. cosas en la estabilidad de las propiedades del 
Figura 1. Triángulo de texturas en el que se representan los resultados de los análisis granulo métricos para las seis comunidades de pastos.

Texture triangle with the results of the granulometric analysis of the six grassland communities.

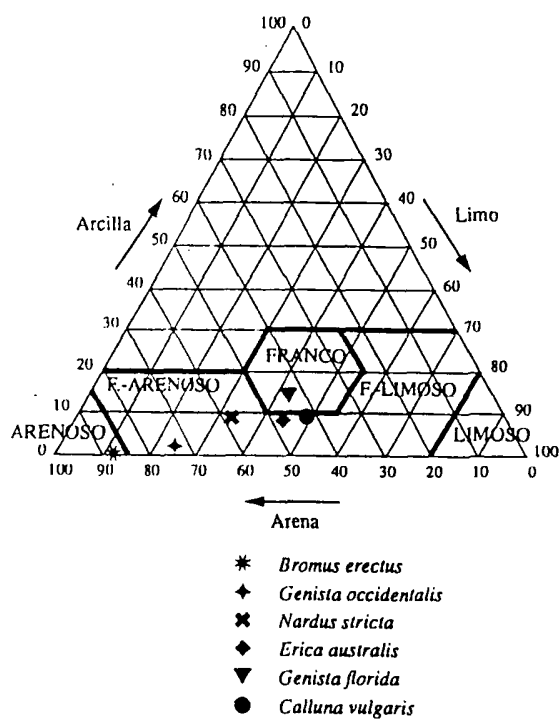

Tabla 3. Análisis de la varianza de los valores medios de la concentración de los elementos analizados en las muestras de vegetación y para cada mes. E.S. = error estándar. SIG $=$ significación. $\mathbf{n}=10$. ANOVA of mean values of the concentration of each analysed element in vegetation samples and each month. E.S. $=$ standard error. SIG = significance. $n=10$

\section{ELEMENTOS}

\begin{tabular}{|c|c|c|c|c|c|c|c|}
\hline & $\mathrm{Ca}$ & $\mathrm{Cu}$ & $\mathrm{Fe}$ & $\mathrm{Mg}$ & $\mathbf{N}$ & K & $\mathrm{Na}$ \\
\hline E. australis & 2894 a & $4.7 \mathrm{a}$ & 97 a & $1370 \mathrm{a}$ & $1.5 \mathrm{a}$ & $9806 \mathrm{ab}$ & 814 a \\
\hline B. erectus & $9356 \mathrm{~b}$ & $7: 0 \mathrm{~b}$ & $206 \mathrm{~b}$ & $2147 \mathrm{~b}$ & $2.0 \mathrm{~b}$ & $10847 b$ & $648 a b$ \\
\hline G. florida & $1824 \mathrm{C}$ & $5.8 \mathrm{c}$ & $185 \mathrm{~b}$ & 2051 bc & $2.0 \mathrm{~b}$ & $10889 \mathrm{~b}$ & $493 \mathrm{~b}$ \\
\hline N. stricta & 3000 a & $5.9 \mathrm{c}$ & $234 \mathrm{~b}$ & $1882 \mathrm{~cd}$ & $2.0 \mathrm{~b}$ & $12720 \mathrm{c}$ & 1069 c \\
\hline G. occidentalis & $7694 \mathrm{~d}$ & $6.9 \mathrm{~b}$ & $120 \mathrm{a}$ & $1628 \mathrm{e}$ & $1.8 \mathrm{~cd}$ & $11078 \mathrm{~b}$ & $513 \mathrm{~b}$ \\
\hline C. vulgaris & $4327 \mathrm{e}$ & $4.3 \mathrm{a}$ & 72 a & $1643 \mathrm{de}$ & $1.7 \mathrm{ad}$ & 9429 a & $657 \mathrm{ab}$ \\
\hline E.S. & 337.90 & 0.29 & 17.54 & 90.43 & 0.23 & 505.08 & 82.81 \\
\hline Junio & $3630 \mathrm{a}$ & $7.7 \mathrm{a}$ & 156 a & 1651 a & $2.1 \mathrm{a}$ & $15 \overline{479}$ a & $765 \mathrm{a}$ \\
\hline Julio & $5823 \mathrm{~b}$ & $6.2 \mathrm{~b}$ & $183 \mathrm{a}$ & $1950 \mathrm{~b}$ & $1.9 \mathrm{~b}$ & $109.00 \mathrm{~b}$ & $509 \mathrm{~b}$ \\
\hline Agosto & $5665 \mathrm{~b}$ & $3.9 \mathrm{c}$ & $115 \mathrm{~b}$ & 1639 a & $1.3 \mathrm{c}$ & $7024 \mathrm{c}$ & 701 a \\
\hline Septiembre & 4279 a & $5.3 \mathrm{~d}$ & 156 a & $1907 \mathrm{~b}$ & $1.9 \mathrm{~b}$ & $9776 \mathrm{~b}$ & 821 a \\
\hline E.S. & 275.89 & 0.23 & 14.32 & 73.83 & 0.19 & 412.40 & 67.61 \\
\hline \multicolumn{8}{|c|}{ Nivel de significación } \\
\hline Com. & $* * *$ & $* * *$ & $* * *$ & $* * *$ & $* * *$ & $* * *$ & $* * *$ \\
\hline Mes & $* * *$ & $* * *$ & ** & $* *$ & $* * *$ & $* * *$ & ** \\
\hline
\end{tabular}

Nivel de significación: ${ }^{* * *}=\mathrm{P}<0.001 ;{ }^{* *}=\mathrm{P}<0.01 ;$ N.S. $=$ no significativo 
suelo, contribuye una vez más a que esta comunidad presente un amplio rango de variación entre las muestras.

Las condiciones edáficas de la comunidad de Genista florida son bastante buenas: la arcilla, humedad, capacidad de retención y materia orgánica son muy altas. Sin embargo, el acúmulo de materia orgánica sobre un substrato silíceo produce una acidificación intensa del suelo. Esta comunidad tiene una producción de alrededor de $3.000 \mathrm{~kg} \mathrm{ha}^{-1}$, que apenas es consumida y las hojas muertas o decadentes se depositan en la superficie del suelo cada año (ALON$\mathrm{SO}, 1994)$. Consecuentemente, el $\mathrm{pH}$ es significativamente más bajo que en las otras comunidades (tabla 1). Este factor, junto al efecto sombra producido por los piornos, da lugar a una flora poco diversa (ALONSO et al., 1993), que conserva sus características químicas durante todo el verano (ver más abajo). Aún así, las plantas que logran desarrollarse lo hacen con gran vigor.

La materia orgánica de los suelos de los pastizales del puerto de San Isidro es mayor que la encontrada por ALVAREZ et al. (1990) en diversos sistemas pastorales de la montaña leonesa, así como en los prados de siega de la Cordillera Cantábrica analizados por GARCIA et al. (1992), debido fundamentalmente al manejo que se aplica en cada zona: mientras que en los prados la biomasa aérea es segada y retirada de la zona, en los pastos la hierba no consumida muere y se descompone contribuyendo a incrementar este parámetro.

\subsection{Fracción mineral edáfica}

Considerando la relativamente pequeña superficie estudiada, se apreció una gran heterogeneidad en la composición mineral de los suelos. Este tipo de variabilidad espacial tiende a ocultar o empequeñecer las variaciones estacionales (ALLEN, 1989), por lo que únicamente se realizó un muestreo de los suelos. La presencia de una alta concentración de calcio, junto a un pH más elevado determina la aparición de una flora diversa, característica de los suelos sobre sustrato calcáreo (ALONSO et al., 1994). En conjunto, los suelos del puerto estudiado tienen niveles normales de $\mathrm{N}, \mathrm{Ca}, \mathrm{Mg}$ y Cu, y bajos de $\mathrm{K}$, Na y Fe cuando se comparan con los resultados de otros autores (BEAR, 1965; FRIED y BROESHART, 1967; DUQUE MACIAS y GONZALEZ CARRENOO, 1972; KNEZEZ y ELLIS, 1980; JONES y THOMAS, 1987; GARCIA GONZALEZ y CLARK, 1989; GARCIA et al., 1992; MORENO et al., 1992).

En la comunidad de Genista florida la concentración de $\mathrm{N}$ es relativamente alta, lo que condiciona la aparición de leguminosas en el pasto: únicamente fueron observadas dos en el pasto, además de las otras dos especies de matorral (ALONSO, 1994). En cuanto a la concentración de otros elementos, es pobre en $\mathrm{K}, \mathrm{Ca}, \mathrm{Mg}$ y $\mathrm{Cu}$, y rica en $\mathrm{Na}$ y $\mathrm{Fe}$. 
Calcio

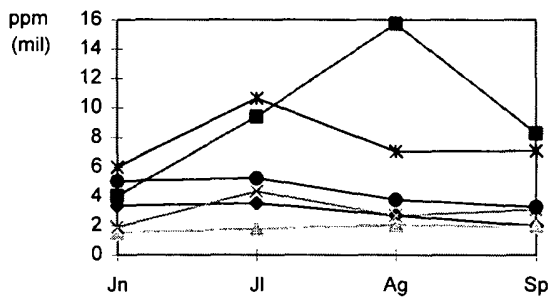

Hierro

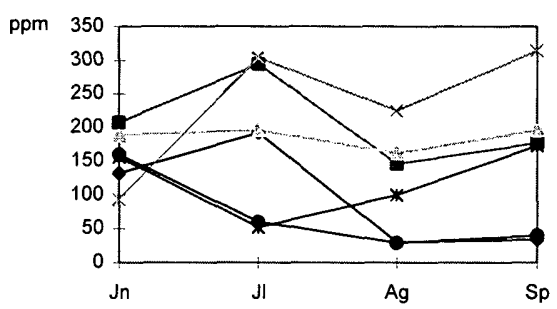

Nitrógeno

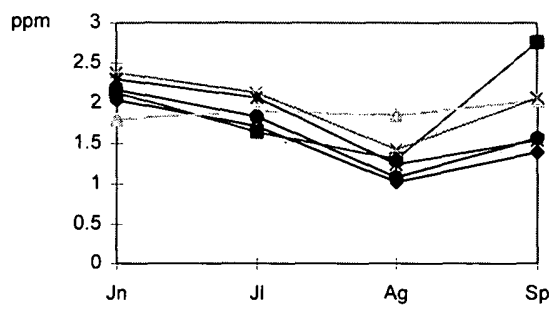

Sodio

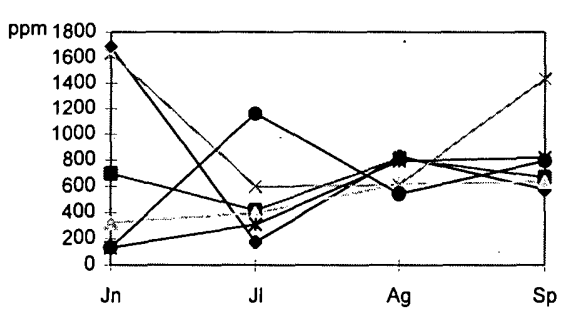

Cobre

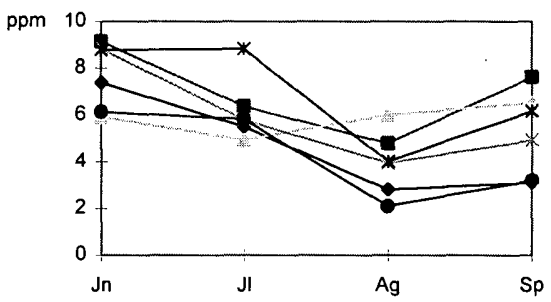

Magnesio

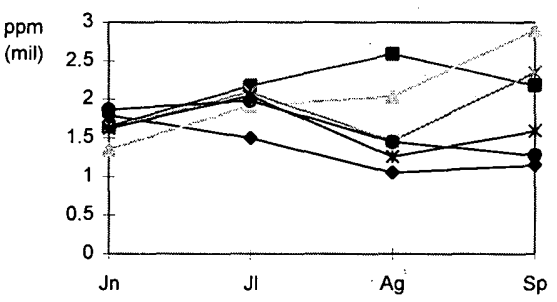

Potasio

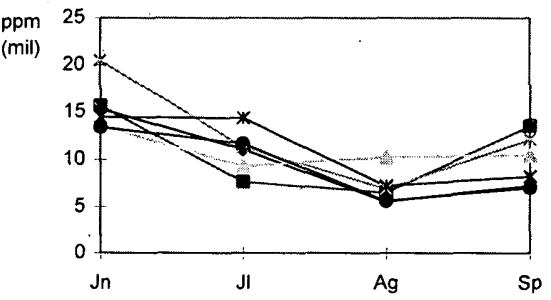

Figura 2. Variación estacional de las concentraciones de los elementos minerales en las muestras de la vegetación de las seis comunidades de pastos.

Seasonal variation of the concentrations of the mineral elements in the vegetation samples in the six grassland communities. 


\subsection{Composición mineral de la vegetación}

La composición mineral de los vegetales no está correlacionada con la composición mineral del suelo (PINTO TOBALINA et al., 1991; ALONSO, 1994). Existen diferencias estacionales en la composición mineral de los pastos para la mayoría de los elementos, a diferencia de datos aportados por otros investigadores para el potasio (BARNES et al., 1990) y de forma similar a los resultados de GARCIA GONZALEZ y ALVERA (1986) y GARCIA et al. (1990). Genista florida muestra de nuevo unas características peculiares, ya que las concentraciones de los elementos apenas mostraron variaciones a lo largo del periodo de estudio.

Considerando las necesidades minerales del ganado ovino y bovino determinadas por GRACE (1983), los pastos de Nardus stricta y Bromus erectus fueron los que mantuvieron el mejor rango de concentraciones necesarias para el mantenimiento de los herbívoros durante el periodo estival. Las muestras analizadas presentan deficiencias importantes de $\mathrm{Ca}$ y $\mathrm{Cu}$, al menos en parte de la estación (agosto) (ALONSO y GARCIA-OLALLA, 1997), siempre según los datos de GRACE (1983). El resto de los elementos se mantiene dentro de un rango de concentración apropiado según los datos indicados por otros autores (MONTALVO et al., 1975; KNEZEZ y ELLIS, 1980; KABATA-PENDIAS y PENDIAS, 1984; GARCIA GONZALEZ y ALVERA, 1986; MARRS et al., 1989; ALVAREZ et al., 1990; GARCIA et al., 1990; McNAUGHTON, 1990; PINTOTO BALINA et al., 1991).

A comienzos de la estación todas las comunidades tuvieron concentraciones superiores a las mínimas indicadas por GRACE (1983). En cambio, durante agosto, excepto Genista florida, todas son deficitarias. En septiembre las comunidades de Bromus erectus, Genista florida y Nardus stricta estarían en condiciones óptimas para el crecimiento vegetal, debido a sus características edáficas, mientras que las otras tres no. $L a$ variación de la concentración de los elementos al transcurrir la estación es debida a la madurez y los cambios fisiológicos (GEORGIADIS y McNAUGHTON, 1990).

$L a$ variación temporal de las concentraciones fue distinta para cada elemento: $\mathrm{K}, \mathrm{Ca}, \mathrm{Mg}$ y Cu disminuyeron sus concentraciones de forma general a lo largo del verano. Las hojas maduras los movilizaron hacia los órganos de reserva. $\mathrm{Na}, \mathrm{Fe}$ y $\mathrm{Ni}$, por otro lado, tienen una progresión irregular. En muchos de los elementos la máxima concentración se dio en julio y agosto como también observaron MONTALVO et al. (1975). GARCIA NAVARRO (1988) apunta también diferencias de concentración a distinta altitud.

La diversidad de especies en la zona, tanto herbáceas como arbustivas, permite al ganado que se mueve libremente seleccionar la dieta de más cali- 
dad entre todo el pasto, ajustando el equilibrio entre los compuestos bromatológicos y los minerales (ALONSO y GARCIA-OLALLA, 1997).

Los resultados conseguidos sugieren que las características edafológicas no parecen limitar el desarrollo vegetal, que podría verse más afectado por la climatología. Los valores obtenidos pueden considerarse normales dentro de los rangos establecidos por otros autores, siendo los suelos sobre substratos calizos los que presentaron las mayores concentraciones de los elementos analizados. La vegetación presentó asimismo valores típicos, excepto en algunas comunidades durante el verano. Sin embargo, la movilidad de los animales les permitiría tener acceso a una dieta variada, seleccionada entre todas las comunidades vegetales presentes. El pastoreo de esta vegetación es deseable para la conservación del ecosistema en las condiciones açtuales, tanto productivas como fisionómicas.

Agradecimientos. Este trabajo fue llevado a cabo gracias a una beca del Plan Nacional de Formación del Personal Investigador del Ministerio de Educación y Ciencia en la Estación Agrícola Experimental del C.S.I.C. en León. Los autores agradecen los comentarios constructivos de las dos personas que revisaron el manuscrito.

\section{Referencias}

ALLEN, S.E. (1989): Chemical Analysis of Ecological Materials. S.E. Allen (Ed.). Blackwell Scientific Publications. Oxford. 368 pp.

ALONSO, I. (1994): Estudio Ecológico y Valoración de un Puerto de la Montaña de León. Tesis Doctoral. Universidad de León. 383 pp.

ALONSO, I.; BERMUDEZ, F.F.; GARCIA, A.; REVESADO, P.R.; MANTECON, A.R.; GONZALEZ, J.S.; CARLOS, G. (1993): Estudio de las comunidades de interés pascícola en un puerto de montaña: I. Estructura y valor pastoral. Pirineos, 141-142:3-18.

ALONSO, I.; GARCIA, A. (1993): Structure of plant communities in a pastoral system in northern Spain. Proc. XVII International Grassland Congress: 324-326.

ALONSO, I; GARCIA-OLALLA, C. 1997. Mineral composition of soils and vegetation from six mountain grassland communities in Northern Spain. Journal of the Science of Food and Agriculture (En prensa).

ALVAREZ, J; PASCUAL, M.R.; CARPINTERO, C. (1990): Estudio de los suelos y la vegetación de prados y pastos de la cuenca del río Porma (León). XXX Reunión Científica de la SEEP. San Sebastián. pp. 23-30. 
BARNES, T.G.; VARNER, L.W.; BLANKENSHIP, L.H.; FILLINGER, T.J. HEINEMAN, S.C. (1990): Macro and trace mineral content of selected south Texas deer forages. Journal of Range Management, 43 (3): 220-223.

BEAR, F.E. (1965). Soils in Relation to Crop Growth. Reinhold Publishing Corporation. New York. 297 pp.

BEESON, K.C.; MATRONE, G. (1976): The Soil Factor in Nutrition Animal and Human. M. Dekker (Ed.). New York. 152 pp.

BEN-SHAHAR, R.; COE, M.J. (1992): The relationships between soil factors, grass nutrients and the foraging behaviour of Wildebeest and zebra. Oecologia (en prensa).

BURRIDGE, J. (1987): Effects of soil conditions on plant trace element uptake and distribution of trace elements in plant parts and species. Annual Report of Macaulay Land Use Research Institute: 64.

DAVIS, B.; WALKER, N.; BALL, D. FITTER, A. (1992): The Soil. The New Naturalist, Harper Collins Publishers. 192 pp.

DE RUIG, W.G. (1986). Atomic absorption spectrometric determination of Calcium, Copper, Iron, Magnesium, Manganese, Potassium, Sodium and Zinc in animal feeding stuffs. Journal of the Association of the Official Analitical Chemist, 69 (6): 1009- 1013.

DUCHAUFOUR, P. (1975): Manual de Edafología. Ed. Toray-Masson, SA. Barcelona.

DUQUE MACIAS, F.; GONZALEZ CARREÑO, T. (1972): Estudio de oligoelementos en suelos de pastos de la provincia de Salamanca. 1. Cobre. Pastos, 2 (2): 228-235.

EGAN, A.R. (1975): The diagnosis of trace element deficiencies in the grazing ruminant. En: Trace elements in Soil-Plant-Animal Systems. D.J.D. Nicholas; A.R. Egan. (Eds.). Academic Press Inc. New York. pp. 371-384.

FRIED, M.; BROESHART, H. (1967): The Soil-Plant System in Relation to Inorganic Nutrition. Academic Press. New York.

GALLARDO, FJ.; CUADRADO, S.; PRAT, L. (1980): Características de los suelos forestales de la Sierra de Gata. Studia Oecologica, 241-264.

GARCIA GONZALEZ, A.; CLARK, S. (1989): The distribution of Minuartia verna and Thlaspi alpestre in the British Isles in relation to 13 soil metals. Vegetatio, 84: 87-98.

GARCIA GONZALEZ, R.; ALVERA, B. (1986): Relaciones entre la composición mineral de las plantas abundantes en pastos supraforestales pirenaicos y su utilización por los rumiantes. XXVI Reunión Científica de la SEEP. Oviedo. pp. 249-265.

GARCIA NAVARRO, R. (1988): Aspectos Agronómicos y Composición Mineral de los Henos, Gramineas, Leguminosas y "Otras Plantas" de Prados Permanentes de la Montaña de León. Tesis Doctoral. Universidad de León. Facultad de Biología. 
GARCIA, A.; PASTOR, J.; BERMUDEZ, F.F. (1988): Valor de los pastos en zonas degradadas de la región central. Avances en Alimentación y Mejora Animal, 28 (2): 57- 61.

GARCIA A.; CARLOS, G.; CHOCARRO, C.; FANLO, R.; FILLAT, F. (1990): Contenido mineral de prados de fondo de valle en la Cordillera Cantábrica y Pirineos. XXX Reunión Científica de la SEEP. San Sebastián. pp. 387-394

GARCIA, A.; CARLOS, G.; BERMUDEZ, F.F. (1992): Vegetation, soil and management relations in meadow communities of the Valdeón Valley, Cordillera Cantábrica, Spain. Coenoses, 7 (1): 55-60.

GEORGIADIS, N.J.; MCNAUGHTON, S.J. (1990): Elemental and fibre contents of Savanna grasses: variation with grazing, soil type, season and species. Journal of Applied Ecology, 27: 623-634.

GRACE, N.D. (Ed). (1983): The mineral requirements of grazing ruminants. New Zealand Society of Animal Production. Occasional Publication No. 9.

JONES, D.I.H.; THOMAS, T.A. (1987): Minerals in pastures and supplements. En: Ecosystemis of the World. 17B. Managed Grasslands, Analytical Studies. R.W. Snaydon (Ed.). Ed. Elsevier. Amsterdam. pp. 145-153.

KABATA-PENDIAS, A.; PENDIAS, H. (1984): Trace Elements in Soils and Plants. CRC Press. Inc. Boca Ratón. Florida.

KINZEL, H. (1983): Influence of limestone, silicates and soil pH on vegetation. En: Physiological Plant Ecology Ill (Encyclopedia of Plant Physiology. New Series 12C). O.L. Lange, P.S. Nobel, C.B. Osmond, H. Ziegier (Eds.). SpringerVerlag. Berlín.

KNEZEZ, B.D.; ELLIS, B.G. (1980): Essential micronutrients IV: Cu, Fe, $\mathrm{Mn}$, Zn. En: Applied Soil Trace Elements. B.E. Davies (Ed.). John Wiley. Chichester.

MARRS, R.H.; RIZAND, A.; HARRISON, A.F. (1989): The effects of removing sheep grazing on soil chemistry above-ground nutrient distribution and selected aspects of soil fertility in long-term experiments at Moor House National Nature Reserve. Journal of Applied Ecology, 26: 647-661.

McNAUGHTON, S.J. (1990): Mineral nutrition and seasonal movements of African migratory ungulates. Nature, 345 (6276): 613-615.

MONTALVO, J., GARCIA, B., GOMEZ, J.M. (1975): Variación de la composición mineral de la hierba de una pradera semiagostante con su estado de madurez. Pastos, 7 (2): 280-295.

MORENO, A.M.; PÉREZ, L.; GONZALEZ, J. (1992): Relaciones entre contenidos totales de $\mathrm{Zn}, \mathrm{Pb}, \mathrm{Cu}$ y $\mathrm{Cd}$ en suelos y plantas. Suelo y Planta (CSIC), 2 (4): 757-771.

PEGTEL, D.M. (1987): Soil fertility and the composition of seminatural grassland. En: Disturbance in Grasslands. J. VAN ANDEL et al., (Eds.), Dr. W. Junk Publishers, Dordrecht. pp. 285-307. 
PINTO TOBALINA, M.; RODRIGUEZ JULIA, M.; DOMINGO URIARTE, M. (1991): Caracterización de las praderas permanentes en el País Vasco. XXXI Reunión Científica de la SEEP. Murcia. pp.123-129.

REVESADO, P.R.; GONZALEZ, J.S.; FRUTOS, P.; RAMOS, G.; ALONSO, I.; GARCIA, A.; BERMUDEZ, F.F. (1993): Estudio de las comunidades de interés pascícola en un puerto de montaña: Il. Evolución en la intensidad de selección del pasto por dos razas ovinas (Churra y Merina). Pirineos, 143-144: 43-53. 